Arts programmes are provided as part of the psychosocial rehabilitation for consumers with a mental illness. Consumers have reported that involvement in the arts has facilitated their recovery. This qualitative study was conducted to explore the ways in which involvement in an Australian community arts programme contributed to the recovery process. It also aimed to gain an understanding of intrinsic recovery, which focuses on personal satisfaction and meaning.

Eight consumers with a mental illness who were members of a community-based arts programme participated in in-depth semi-structured interviews. The themes that were analysed thematically from the interviews included art as a medium of expression and self-discovery, and changes in internal conditions (spirituality, empowerment and self-validation). The findings revealed the usefulness of arts programmes in providing the external environment that facilitates internal recovery.

\title{
Art and Recovery in Mental Health: a Qualitative Investigation
}

\author{
Chris Lloyd, Su Ren Wong ${ }^{1,2}$ and Leon Petchkovsky ${ }^{1}$
}

\section{Introduction}

Recovery is a journey towards a new and valued sense of identity, role and purpose, beyond the diagnosis of a mental illness. It involves living well despite any limitations that may be imposed by the illness (Queensland Health 2005). People with mental illness have found recovery to be a subjective and individualised process. Unlike the biomedical definition of recovery, it is not a predictable and medically mediated prognosis but, rather, the emergence of a new identity as a product of self-discovery (Deegan 2001).

Recently, this perspective of recovery has influenced the provision of mental health services. Such services are directed towards motivating individuals by providing opportunities for self-exploration and the development of their interests, instead of curing them back into so-called 'normality' (Jacobson and Greenley 2001). As a result, community arts programmes have been developed as a means of providing a platform for exploration.

${ }^{1}$ University of Queensland. ${ }^{2}$ National University Hospital, Singapore.

Corresponding author: Dr Chris Lloyd, Senior Lecturer, Division of Occupational Therapy, School of Health and Rehabilitation Sciences, University of Queensland, St Lucia, Q 4072, Australia.

Email: Iloyd@onthenet.com.au

Submitted: 15 August 2006.

Accepted: 23 April 2007.

Key words: Mental health, recovery, community arts.

Reference: Lloyd C, Wong SR, Petchkovsky L (2007) Art and recovery in mental health: a qualitative investigation. British Journal of Occupational Therapy, 70(5), 207-214.
Although believed to be supportive of recovery, the effectiveness of such programmes is not supported strongly by the literature. The purpose of this study was therefore to explore the ways in which involvement in an arts programme contributed to the recovery process of individuals with mental illness.

\section{Literature review}

\section{The arts in mental health}

The arts have had a long connection with mental health. First introduced as art therapy, art was used in diagnosis and projection therapy without regarding the aesthetic aspects (Miller 1998). A newer variation, known as the 'therapeutic use of art', focused on the therapeutic relationship between therapist and consumer, the process of creating art and communication through art (Lloyd and Papas 1999). Mental health rehabilitation services that are integrated into the community are reported better to facilitate the recovery process because they 'provide normalisation experiences that mimic naturally occurring niches' (Miller 1998, p169). Various examples include Splash Art Studio (Miller 1998) and The Artful Dodger's Studio (Thiele and Marsden 2003) in Melbourne and Girrebala (Lloyd and Chandler 1999a, 1999b) on the Gold Coast in Australia. Arts programmes have also been described in Scotland, for example Islay and Jura Art Therapy, Mid-Argyll Community Mental Health Team Art Therapy and Midlothian Art Therapy (Mental Health Foundation 2006). 


\section{The recovery model}

The 'recovery model' emerged in psychosocial rehabilitation in the 1990s (Anthony 1993). It replaced the biomedical foundations of rehabilitation with a subjective experience called 'recovery'. Recovery is defined as a uniquely subjective process that includes both an external and an internal change of conditions (Jacobson and Greenley 2001). Concepts of internal change, such as increased autonomy and the ability to accept change and risks as well as the development of a new identity, seem to be experienced commonly (Murphy 1998, Bledsoe 2001, Deegan 2001, Pierce 2004).

Jacobson and Greenley (2001) developed a conceptual model of recovery, which included four intrinsic conditions and three environmental conditions. Hope is the belief that recovery is achievable. A sense of hope is found through spirituality or other means, including participation in art. Hope involves not only accepting the difficulties of mental illness but also focusing on the strengths of the person and the positive opportunities in life. Healing is not the same as defined in the medical model. Rather than 'a return to "normal" health', it is about discovering a new identity apart from the illness and gaining a sense of 'control' over the symptoms of the illness (Jacobson and Greenley 2001, p483).

Empowerment is the perception that a person has choices and control over the consequences in his or her life. Connection includes the formation of social connections with others and the sense of belonging and contribution to the community.

\section{Girrebala arts programme}

The Girrebala arts programme on the Gold Coast, Australia, was selected for this study. All members of Girrebala are registered clients of the Gold Coast Mental Health Service. Conducted by an artist-in-residence and an occupational therapist, a community arts programme is provided for consumers with a serious mental illness. The programmes are organised in 10-week blocks. People can re-engage in programmes for as long as they feel the need to be involved in developing their artistic talents. The groups cater to different levels of interest, which explore various media and techniques of creating art, for example, watercolours, oils and screen printing. A major part of the involvement is participation in an annual art exhibition. Each exhibition showcases certain artists, called 'profile artists'. Their work is judged by a local art critic and they receive awards for work that meets the judging criteria. All works are for sale during the exhibition.

According to written accounts, Girrebala is a supportive environment for recovery (Lloyd and Chandler 1999a, 1999b). Providing the programme in the community increases accessibility and decreases the stigma associated with hospital-based services. Focusing on artistic competencies, instead of disability, helps to encourage self-esteem and thus facilitates the formation of a new identity (that is, healing). The programme aims at empowering consumers to achieve their artistic goals.

\section{Method}

\section{Study design}

A qualitative method was chosen, focusing on personal experiences. It followed the assumption that reality is what people experience it to be and thus the individual is 'the originator of meaning and the central point for social analysis' (Grbich 1998, p167). In keeping with the subjectivity of the recovery model, this method emphasised not only what individuals experienced but also how it was meaningful to them. According to Patton (1998), the world is first perceived through our senses and then expressed through our understanding of reality. Therefore, the description (objectivity) and interpretations (subjectivity) of the participants' experiences cannot be separated. Similarly, the analysis of the data should be investigated as a whole. Biases are not minimised but are analysed critically as part of the data.

\section{Participants}

The individuals with more than one year's experience in the Girrebala arts programme were approached by the artist-in-residence, who explained the study to them. It was important for them to have been involved for more than a year so that they would have experienced the various major events of the programme. No more than the first 8 to 10 individuals who agreed to participate were to be interviewed. If they volunteered to participate, formal written information was provided and their written consent was obtained. The participants were able to volunteer to participate of their own free will. They were assured that they had the right to withdraw at any time and that their subsequent treatment would not be affected in any way. A profile of the participants is provided in Table 1.

Approval was granted for this study from appropriate ethical committees at the Gold Coast Hospital and the University of Queensland.

\section{Data collection procedure}

Semi-structured interviews were used to capture the depth of the individual's recovery experiences. Semi-structured interviews provided the freedom to explore the experience of art and recovery while at the same time providing some structure to remain focused (see Appendix 1). Part of the interview involved the use of visual material, which allowed the person to express himself or herself more than what was said (Mason 2002). The interviews were held at the Girrebala house, where the participants reportedly felt comfortable and spontaneous in disclosing their experiences. The interviews were conducted by a supervising consultant psychiatrist and the principal researcher. Each interview lasted approximately 50-90 minutes, depending on the amount of disclosure with which the interviewee was comfortable.

Data and investigator triangulation were implemented to increase rigour. All the interviews were both tape-recorded and video-recorded to increase rigour in transcription and observation of what the person was 


\begin{tabular}{|c|c|c|c|c|c|c|}
\hline Participant & Diagnosis & Age range & Marital status & Occupation & Psychotherapy & Medication \\
\hline A... & ...Bipolar affective disorder.. & $\ldots 20-24$. & ...Single.. & ...Student ........... & ... Yes. & ..No ... \\
\hline & ....Schizophrenia ......................... & ....35-39.. & .... Single. & ...Unemployed... &..$Y$ & .. Yes. \\
\hline & ....Bipolar affective disorder.. & $\ldots .25-29$. & ...Single.. & ...Student. & ...Yes & ...Yes ... \\
\hline D.. & ....Depression... & $\ldots 55-59$. & ....Divorced. & ...Student & ...No & ...Yes ... \\
\hline & ....Schizoaffective disorder.. & $\ldots 55-59 .$. & ....Married. & ...Homemaker.. & ...Yes & ....No... \\
\hline F....... & ..Panic disorder ................... & $\ldots .45-49 \ldots$ & ......Married ........ & ...Diversional ther & ..........Yes .... & ...No...... \\
\hline G................. & ....Bipolar affective disorder.. & $\ldots .40-44 \ldots .$. & ......Separated..... & ...Airbrush design & ....................... & ......Yes ... \\
\hline 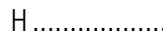 & ...Dysthymic disorder ... & ...35-39. & ...Single... & ...Unemployed.. & ...No... & ...Yes ... \\
\hline
\end{tabular}

describing and explaining (Seidman 1991). Field notes were taken during and after the interview by both the supervisor and the principal researcher, which increased rigour further by gaining multiple perspectives on the observations of the person and the interviewer's judgements and experience (Mason 2002). Following the interview, the interviewers discussed their observations and reflections to reduce undue misinterpretations. Throughout the process of data analysis, data and investigator triangulation were used to decrease inconsistencies (Patton 2002). Copies of the drafts were also available for participant and colleague checks for verification of accuracy.

\section{Data analysis}

The process for thematic data analysis followed the guidelines by Braun and Clarke (2006). This involved the researchers familiarising themselves with the data; generating initial codes; searching for themes; reviewing themes; defining and naming themes; and producing the report. The interviews were first transcribed from the audiotapes and videotapes and analysis was carried out. The transcripts were analysed the first time to identify the set of themes that were present and initial code names were assigned to them (Minichiello et al 1990, Braun and Clarke 2006). With subsequent readings, the transcripts were then systematically coded manually. This phase, which refocused the analysis at the broader level of themes rather than codes, involved sorting the different codes into potential themes and collating all the relevant coded data extracts within the identified themes (Braun and Clarke 2006). This phase ended with a collection of candidate themes and subthemes, and all extracts of data that had been coded in relation to them.

The next step was to review and refine the themes. All the collated extracts for each theme were read. At this stage, the validity of the individual themes was considered in relation to the data set and whether the candidate themes accurately reflected the meanings evident in the data set as a whole. The data set was reread to ascertain whether the themes worked in relation to the data set and to code any additional data within the themes that had been missed in earlier coding stages (Braun and Clarke 2006). Finally, the essence of what each theme was about was identified and the aspect of the data that each theme captured was determined. The collated data extracts for each theme were then returned to and organised into a coherent and internally consistent account, with an accompanying narrative.

\section{Findings and discussion}

\section{Themes}

Five major themes and 15 subthemes were identified, as specified in Table 2. Appropriate quotations from the interviewees have been provided to illustrate the themes. The recovery model was used as a general framework for discussion (Jacobson and Greenley 2001). Therefore, the themes were divided into two main constituents: art as a medium and internal conditions (see Table 2). 'Art as a medium' included the themes that related to the process of manipulation and experimentation with art, during which the internal conditions changed. 'Internal conditions' included the themes referring to personal experiences of recovery.

\section{Art as a medium}

\section{Expression}

The participants found art to be one of their most preferred forms of expression. Art provided a majority of the participants with a way consciously to access an

Table 2. Major themes with respective subthemes

\begin{tabular}{|c|c|c|}
\hline Main constituent & Major theme & Subthemes \\
\hline \multirow[t]{5}{*}{ Art as a medium... } & ... Expression...... & ..Catharsis............ \\
\hline & & Imagination..... \\
\hline & & Survival strategy ................. \\
\hline & Self-discovery... & ..Journey .... \\
\hline & & Specific turning points .... \\
\hline \multirow[t]{10}{*}{ Internal conditions.. } &.. Spirituality...... & ..Meaning and purpose..... \\
\hline & & Acceptance and hope..... \\
\hline & & Purposeful activity ........ \\
\hline & Empowerment . & ..Sense of control................... \\
\hline & & Power to influence........... \\
\hline & Self-validation.. & Sense of identity................. \\
\hline & & Normality....... \\
\hline & & Self-confidence....... \\
\hline & & Self-satisfaction ................. \\
\hline & & Pursuing goals.................... \\
\hline
\end{tabular}


'imaginary world' that was personally very real. Art was a creative way to describe and 'bring it into the world as a physical manifestation of their observing it' (Participant G). The themes of their artwork went beyond their illness and did not reflect just their struggles but also their fantasies and dreams (Kivnick and Erikson 1983).

Art was also useful to access parts of the individual beyond his or her rational thinking and to bring these subconscious emotions to the surface (Anderson 1995), also known to artists as 'surrealism'. Creativity is an important skill in daily life because it is crucial in problem solving (Kivnick and Erikson 1983, Smitskamp 1995). Art is useful in developing creative problem solving through experimenting with materials, because the artist brings into reality something that is new and original (Thiele and Marsden 2003). It is a safe medium in which to manipulate ideas until they are adapted to become an appropriate expression of what the artist wants to articulate.

It was evident that some participants found art to be an effective self-generated strategy in managing their mental illness. The participants with mood disorders mentioned that expressing their thoughts and emotions was essential in surviving and 'conquering' symptoms of mental illness, such as suicidal intents and fears. In this sense, expression through art became a necessary coping strategy for them:

Fortunately, doing art provides that substitute for an actual attempt of suicide. It's a good enough substitute to get relief for some reason. (Participant D.)

\section{Self-discovery}

Although a few participants appreciated learning and mastering new artistic techniques, the majority of the participants expressed that the most meaningful part was gaining 'insight' about themselves. They gained new understandings about themselves, their relationships and the illness. Participant $G$ stated that art was a way to 'communicate with himself on a level that is productive'. Most participants found recovery to be a steady continuous process, likened to a journey:

I think it's been a process. Obviously, I've been coming here consistently for the last four or five years. So, it's always been there and it's been something that's been a second home to me. So I've always felt comfortable here and relating to people and talking about art and enjoying the company and that sort of thing. So, it's never been a one-off sort of thing; it's always been a constant continual sort of thing. (Participant H.)

The concept of a 'journey' or process has been a common experience for most people and their recovery (Bledsoe 2001, Deegan 2001, Mack 2001). Although for most participants in this study recovery appeared to be a gradual process, a few individuals found that there were specific artworks, events or relationships within the programme that were particularly significant points in their recovery process:
I'll probably start off with this photo here - Man of the Underground. And it's a portrait that I did probably four years ago. And it's probably the first painting that I had ever done in such emotion and yeah, it was probably a bit of a pinnacle at the time ... it made me realise what I was capable of in a sort of expression context ... Yeah it did represent me because I was feeling a bit down at the time and I needed art to help me sort of come out of that shell. (Participant A.)

Personal accounts of recovery have reflected a continuous process of discovery, in which one is constantly learning and hopeful of learning (Mack 2001, Deegan 2003, Pierce 2004). This experience appeared to be unique. Some participants focused more on learning specific artistic techniques while allowing the internal processes to adapt subconsciously; others actively wanted to be aware of their internal processes and chose to use art as a reflective process. Both ways appeared to be just as effective in facilitating recovery, bringing about changes in their internal conditions.

\section{Internal conditions}

\section{Spirituality}

Although not always explicitly stated, it appeared that most participants found that there was a connection between art and the core of their existence, also known as 'spirituality' (Deegan 2001):

I mean art is a very interesting thing, it's life for me, you know. It's something very spiritual you know. It's not always one answer; it's always different things like you know, you might finish one artwork and that's it. And you might finish another artwork and it might be 'Hold on a second, I can take something out of that and put it in another artwork' or maybe 'I can grow from that'. (Participant A.)

Spirituality has been defined as 'having a belief in a higher being', 'a sense of meaning and purpose', 'connectedness to nature and humanity' and 'altruism' (Hassed 2002). Having a spiritual perspective on life is a protective factor against psychiatric disorders and symptoms. Consumers may turn to other spiritually oriented movements if they sense a gap between their spiritual needs and the care provided by mainstream psychiatry (Hassed 2002). This is especially important for individuals who may not have other means of gaining this fulfilment through religion or other spiritual movements. From the participants' comments, the creation of art appeared to offer a means of gaining spiritual fulfilment by providing meaning and purpose as well as a way to relate to a higher power. Certain major spiritual constructs that emerged in the interviews (meaning, purpose, acceptance and hope) will be further elaborated.

\section{Meaning and purpose}

The participants reported art as being an activity that was both meaningful and purposeful for them. It was personally meaningful because it was new, interesting, fulfilling and applicable to their lives: 
So because on another level, some people don't understand why I spend time in my garage painting pictures and they want to challenge that and say I should be washing dishes at the fish and chips shop. And like ultimately, I don't really know why I do what I do but I'm happy doing it and I don't see any need to change what works for me. (Participant G.)

The pieces served as milestones in their recovery, because it was natural for them to engage in art during challenging times. The pieces were likened to photographs, which captured the essence of their experience at that point in time. Often, the impression of meaning involved in the art was a subconscious effort and the artists only realised the significance further along their recovery journey:

So, like this painting here in particular, I did this one at the time when my marriage was sort of breaking up and that ... And at the time I paint these, I don't really reflect on the meaning. It's only after that, that I sort of get a look at what I painted and sort of reflect on it. (Participant G.)

A majority of the participants found that art was not only meaningful but also purposeful. They found that art was something that they wanted to continue to pursue. Many found purpose in pursuing artistic excellence, as well as communicating messages through their work that might sometimes be 'controversial' because they challenged society's perceptions and views:

This is my mission to research, to look into issues of culture, human culture from the perspective of my own human experience. And that is basically what artists do and use art as a means of researching, commenting and documenting and communicating to people ... To influence to show that mental illness is not something unusual or being normal is not normal either. So we are not as a mental patient, we are not that necessarily different. (Participant D.)

As shown above, it seems that artists often see themselves as social and cultural critics (Thiele and Marsden 2003), finding meaning and purpose in sharing their opinions about the human condition.

\section{Acceptance and hope}

Most participants did not perceive recovery as returning to their previous level of function, but accepted their illness as part of them for the rest of their lives; they believed that recovery was about managing their illness more than removing it (Pierce 2004). The acceptance of the illness also led to the acceptance of a new lifestyle, with new aims and goals. Whether or not the participants hoped that they could recover from their illness, art gave them a hope for their future. Hope draws upon past and present experiences and assures the individual of possibilities in the future (Murphy 1998, Jacobson and Greenley 2001). With the positive results that they had seen from their involvement in Girrebala, the participants anticipated a hope of achieving future personal accomplishments in, for example, further studies or employment:
I hope to attain the credentials I need to get into that and I know what it will require you know and in terms of an OP and everything so I hope this course will allow me to gain access to the main degree but I won't necessarily continue straight away with it. (Participant C.)

Although the participants struggled to engage in their art consistently, the motivation to persevere was present because of their hope of recovery or what possibilities lay ahead.

\section{Empowerment}

\section{Sense of control}

Engaging in art helped the participants to regain a sense of control (Jacobson and Greenley 2001). Acute episodes of mental illness appear to be markers of a person's perception of an internal loss of control (McGruder 2001). The stigma accompanying diagnosis has also been identified as a precipitating factor (Davidson et al 2001). The participants felt empowered to make choices about the outcome of their artwork. They could choose if they wanted to express everything in their minds or to keep some things coded and symbolic, such that only they could decode the true meaning behind their artwork:

Initially, I used to re-enact the suicide attempts by drawing disguised figures, positioning them in a corner so you could always say there is no rope but the corner of a room was done in such a way that suggested a rope. I did a lot of double interpretation, imagery, so I could always say, safely say, that it's not but the symbolics was there for me for that particular purpose. (Participant D.)

As the participants felt empowered over the smaller situations, they were also able to generalise this sense of control to other life situations. The element of empowerment is identified as essential for recovery (Barker 1999, Jacobson and Greenley 2001). It is the basis of increased independence, including a boldness to take calculated risks and to accept the consequences of these risks. The participants did not always perceive themselves as at the pinnacle of these abilities, but as growing within them. Their accounts showed the different stages of the struggle that they had with self-preservation and taking risks but also reflected a desire to balance both:

And at the moment, I'm on my guard, like I said to you, I'm on my guard, I'm protective of me because I'm the best I've ever been. But it's accelerated since I found art. (Participant F.)

The participants began to accept responsibility for the consequences of their actions instead of blaming it on others (Jacobson and Greenley 2001). A majority of the participants found that art helped them to overcome the fear of scrutiny that is often associated with risk. They learnt to be less sensitive of criticism about their artwork, seeing it instead as positive feedback (Thiele and Marsden 2003).

\section{Power to influence}

The participants felt that they could not only control the circumstances in their lives but also influence the lives of 
others. They reported decreased instances of hospitalisation after being part of the arts programme. This led to less disruption of daily life, not just for themselves but also for their families (Bledsoe 2001). The perceived value of their contribution to society also increased as they experienced an increased ability to influence their surroundings. A majority of the participants felt that, through their art, they had an impact on those who bought their pieces, and found it satisfying to be a creator of something that was useful and meaningful and brought enjoyment to others in society:

And they were just impressed with the three pieces I had, which were only the beginning, which I regret now that I've sold. But they sold! The response to my type of art for therapy must have attached itself to somebody who wanted to buy it and keep it. (Participant F.)

As shown above and in other accounts of individuals recovering from mental illness, the participants valued the need to be useful and successful (Bledsoe 2001, Davidson et al 2001). Art was a medium in which they could communicate issues that were important to them through socially acceptable means and thus reach a larger audience in a non-threatening way for both the artist and the community. Accomplishing a piece of artwork that contains images and concepts that can have an impact on another person is very empowering (Thiele and Marsden 2003).

\section{Self-validation}

\section{Sense of identity}

The issue of identity is quite critical - one of the experiences of a mental illness or reaction to a mental illness is associated with a loss of identity. (Participant D.)

After being diagnosed with mental illness, many consumers feel their identity overwhelmed by the stigma of the illness (Murphy 1998, Deegan 2001). Although there is an increased awareness of mental illness in society, many participants in this study still expressed the feeling of being reduced to the label of their diagnosis (Deegan 1993, Jacobson and Greenley 2001).

Art had a major role in piecing together a new identity for oneself. The participants felt that creating art assisted them in coming to a realisation that the illness was a manageable part of their lives and did not inevitably overwhelm their personality. The formation of a new identity is a common experience for others in recovery (Deegan 1993, Bledsoe 2001, Mack 2001): 'Recovery enables me to have options and choices within these roles. It goes way beyond my mental illness. It has opened pathways for opportunities and new discoveries' (Bledsoe 2001, p36).

The basis of a stronger sense of identity came from the formation of a new belief system throughout the process of art creation. A majority of the participants expressed a new belief of 'normality' and how they had come to believe that they were normal in their own right:
I don't separate myself from anyone else 'cos that can once, create like a - or isolate myself maybe 'cos I'm healthy but you create all kinds of conditions where you are once separated from everyone and that to me is a sign of sickness ... I know I'm just like everyone else but just varies, just a lot more erratic that's all. (Participant C.)

The participants found that art gave them a sense of worth and value. They found assurance in the realisation that normality included a wide spectrum of individuals, with different experiences and thought processes. During the acute stages of their mental illness, they felt the need to return to becoming a so-called normal person.

However, they later found that they did not necessarily want to return to it, but even preferred being the way they were with their new lifestyles and new identities:

I find it almost inconceivable to go back to what I used to do. It's just, it would not work for me. I already find myself in new lines and this is the way I am and I will be in the near future. So any attempt to make me so-called normal would probably not work and prevent me from expressing myself the way I only know; that is through art, through my thoughts. (Participant D.)

Jamieson (1993, p241) stated: 'Many artists and writers believe that turmoil, suffering, and extremes in emotional experience are integral not only to the human condition but to their abilities as artists.' Other accounts of individuals with mental illness also show that it can at times be a pleasurable experience and that they may not necessarily want to lose all the symptoms of their illness (McGruder 2001, McLean 2003). This appeared to be the case for a majority of the participants, because many now found it beneficial to use their experience in creating art instead of suppressing the symptoms.

\section{Self-confidence}

Being part of the arts programme appeared to be helpful in creating a healthy belief in oneself owing to the value placed on the person as an individual, and also to the appreciation of one's accomplishments. Society often associates a person's worth with what he or she can or cannot do, although they are not synonymous. When the participants were able to do something that they could not do before, this increased their self-confidence. The participants felt that being able to complete and showcase a piece of artwork validated them as someone who was useful and valuable:

And that's the reward I suppose and gives yourself a feeling of worth or you can think, oh well, in terms of what people think of you, well maybe at least you can accomplish that, giving yourself, you know, a sense of worth. That's what we always like. (Participant C.)

During the course of discussing their accomplishments, the pleasure and satisfaction expressed in their tone of voice and positive facial expressions were evident:

And when I looked at it; it was a picture and I was so excited! And I ran out and I said to the nurse: 'Come and 
look at this! Come and have a look at this!' ... And I said, 'What are we going to do with this? We can't move it! It's important! Look what I have done!' (Participant F.)

The confidence that comes from overcoming smaller challenges in each art piece created builds up. This is reflected in a more positive verbal and non-verbal communication in general (Thiele and Marsden 2003). The participants that used to be shy and unable to maintain eye contact found that they had become more confident in the way that they communicated.

\section{New lifestyle}

As the internal conditions changed, the participants found their behaviour changing, leading to a new lifestyle. As life became more meaningful, hopeful and purposeful, the participants were encouraged automatically to restructure their cognitive processes towards tasks. With the acceptance of their illness and the hope of recovery, the participants were more willing to search for the most effective way of managing their illness and initiating their own coping strategies:

I'm learning 'cos that is the only way I cope with life, to adapt to what is given to me right here and now, not what's gonna happen tomorrow, 'cos I can't afford to think in terms of past and future, because of the nature of the confusion that comes from it. So, I'm almost trying to live in the safety of the moment when I paint. (Participant C.)

By identifying themselves apart from their illness, they were able to structure and organise their time more effectively in order to reach their goals. The programme provided a framework in which the participants were encouraged to keep active and oriented towards the future. They appreciated the weekly routine, such as the groups and individual work time, as well as having a larger goal to work towards, such as the annual art exhibition: 'The need to keep pretty busy, to keep yourself active is very helpful' (Participant $\mathrm{H}$ ).

Self-confidence and empowerment are essential in positive decision making. Eventually, the participants became confident enough to attempt larger emotional, mental and physical challenges, ultimately overcoming mental illness: 'There's nothing that you can't achieve when you put your mind to it' (Participant G). As a result, a majority of the participants have moved on to take on employment or pursue their interest in art at a tertiary level:

And I steadily regained confidence in doing more and more courses and now I'm studying two days a week in university - X university full-time course. (Participant D.)

The participants found themselves engaging in new life roles, such as an employee or a student, as well as performing their current roles more effectively, such as a spouse or a parent. They felt empowered and equipped to face new challenges in these roles because they were developing the skills to make decisions and take risks.

\section{Limitations of the study and future research}

This qualitative study reflects the individualised subjective experiences of a small group of individuals in relation to participating in a specific community arts programme. It cannot therefore be generalised to all arts programmes per se, but it will be useful in comparing whether such recovery-oriented outcomes are found in similar arts programmes. The real strength of this research is the rich descriptions about the personal value of art for individuals' recovery. A major limitation of the study involved the sampling strategy. It is likely that the first eight to agree to participate were the ones who were particularly appreciative of the benefits. Perhaps there were others who could have been approached who would have been less positive. Further research should be conducted in this area to strengthen the knowledge base of the use of community arts programmes in promoting recovery and community participation.

\section{Conclusion}

This study has explored how involvement in a community arts programme, such as Girrebala, has facilitated internal recovery. The findings indicated that both the creation of art and involvement in that particular programme helped to facilitate internal changes of recovery, as outlined in the conceptual model of recovery. The findings showed that the individual experiences of recovery were similar to those of other previous accounts of recovery.

The participants were encouraged by personal self-discovery and self-generated strategies as a result of internal changes. Ultimately, the consumers' journeys of recovery are all uniquely individual. Each participant expressed the internal changes in different ways, although they may have experienced similar changes. Supportive relationships and the physical environment were found to be significant in providing a creative environment in which they felt safe to take risks and to be vulnerable in their journey of discovery. As more individuals share their experience of recovery in regard to art, there will be a greater understanding of the value of arts programmes in providing opportunities for healing, self-rediscovery, the formation of a new identity, and participation and acceptance in the wider community.

\section{Acknowledgements}

The researchers would like gratefully to acknowledge the generosity of the artists in sharing their experiences with us. We would also like to thank Linda Chandler, the artist-in-residence at Girrebala, who assisted with the arrangements of the interviews, as well as Walter Petersen for filming the interviews. This article is based on a research report, submitted by the second author as a partial requirement for an Honours degree in Occupational Therapy at the University of Queensland, Australia. A version of this paper was presented at the Mental Health Services Conference of Australia and New Zealand in Adelaide, 2005.

\section{References}

Anderson F (1995) Catharsis and empowerment through group claywork with incest survivors. Arts in Psychotherapy, 22, 413-27. 
Anthony W (1993) Recovery from mental illness: the guiding vision of the mental health service system in the 1990s. Psychosocial Rehabilitation Journal, 16, 11-23.

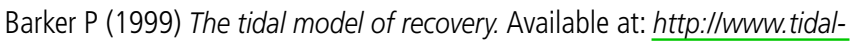
model.co.uk/New\%20intro.htm Accessed on 11.10.04.

Bledsoe C (2001) Unique eyes and different windows of opportunity: the consumer provider perspective. In: C Brown, ed. Recovery and wellness: models of hope and empowerment for people with mental illness. New York: Haworth Press, 23-42.

Braun V, Clarke V (2006). Using thematic analysis in psychology. Qualitative Research in Psychology, 3, 77-101.

Davidson L, Stayner D, Nickou C, Styron T, Rowe M, Chinaman M (2001) 'Simply to be let in': inclusion as a basis for recovery. Psychiatric Rehabilitation Journal, 24(4), 375-88.

Deegan G (2003) Discovering recovery. Psychiatric Rehabilitation Journal, 26(4), 368-76.

Deegan $P$ (1993) Recovering our sense of value after being labeled mentally ill. Journal of Psychosocial Nursing, 31(1), 7-11.

Deegan P (2001) Recovery as a self-directed process of healing and transformation. In: C Brown, ed. Recovery and wellness: models of hope and empowerment for people with mental illness. New York: Haworth Press, 5-21.

Grbich C (1998) Qualitative research in health: an introduction. Sydney: Allen and Unwin.

Hassed C (2002) Are we approaching mental health in the right spirit? Auseinetter, 2, 10-12.

Jacobson N, Greenley D (2001) What is recovery? A conceptual model and explication. Psychiatric Services, 52(4), 482-85.

Jamieson KR (1993) Touched with fire. New York: Simon and Schuster. Kivnick HQ, Erikson JM (1983) The arts as healing. American Journal of Orthopsychiatry, 53, 602-18.

Lloyd C, Chandler L (1999a) Girrebala and the arts in mental health rehabilitation. British Journal of Therapy and Rehabilitation, 6(4), 164-70.

Lloyd C, Chandler L (1999b) Girrebala: an Australian rehabilitation programme. Psychiatric Rehabilitation Skills, 3(2), 173-92.

Lloyd C, Papas V (1999) Art as therapy within occupational therapy in mental health settings. British Journal of Occupational Therapy, 62(1), 31-35.

Mack S (2001) Where the rainbow speaks and catches the sun: an occupational therapist discovers her true colors. In: C Brown, ed. Recovery and wellness: models of hope and empowerment for people with mental illness. New York: Haworth Press, 43-58.

Mason J (2002) Qualitative researching. 2nd ed. London: Sage.

McGruder J (2001) Life experience is not a disease or why medicalising madness is counterproductive to recovery. In: C Brown, ed. Recovery and wellness: models of hope and empowerment for people with mental illness. New York: Haworth Press, 59-80.

McLean R (2003) Recovered, not cured: a journey through schizophrenia. Sydney: Allen and Unwin.

Mental Health Foundation (2006) Arts, creativity and mental health initiative. Glasgow: Mental Health Foundation.

Minichiello V, Aroni R, Timewell E, Alexander L (1990) In-depth interviewing: researching people. Sydney: Longman Cheshire.

Miller A (1998) The role of community arts in psychosocial rehabilitation. In: VICSERV conference papers. Melbourne: Psychiatric Disability Services of Victoria, 167-70.

Murphy M (1998) Rejection, stigma, and hope. Psychiatric Rehabilitation Journal, 22(2), 185-88.
Patton MQ (1998) Qualitative evaluation and research methods. 2nd ed. Thousand Oaks, CA: Sage.

Patton MQ (2002) Qualitative research and evaluation methods. 3rd ed. Thousand Oaks, CA: Sage.

Pierce R (2004) A narrative of hope. Psychiatric Rehabilitation Journal, 27(4), 403-409.

Queensland Health (2005) Sharing responsibility for recovery: creating and sustaining recovery oriented systems of care for mental health. Brisbane: Queensland Health.

Seidman IE (1991) Interviewing as qualitative research: a guide for researchers in education and the social sciences. New York: Teachers College Press.

Smitskamp H (1995) The problem of professional diagnosis in the arts therapies. Arts in Psychotherapy, 22, 181-87.

Thiele M, Marsden S (2003) Engaging art: the Artful Dodger's Studio, a theoretical model of practice. Melbourne: Jesuit Social Services.

\section{Appendix 1. Interview schedule}

- Participation in the arts programme

- How much have you been involved in the Girrebala arts programmme?

- How long?

- How many times a week?

- What kinds of activities have you been involved in as part of this programme?

- Art exhibitions?

- Profile of an artist?

- Self-expression?

- What kinds of things do you do at Girrebala?

- Complete projects?

- Socialise with others?

- Learn new skills/techniques?

- In what ways is this programme similar or different to other services in the mental health system?

- Sense of belonging in the community?

- Structure?

- Interest and motivation?

- Impact of the programme on the person

- In what ways has involvement in the arts programme affected you personally?

- Helps you feel better about yourself (self-esteem)?

- Emotionally (catharsis)?

- Spiritually (hope)?

- Physically (activity)?

- Intellectually/mentally (imagination, concentration, empowerment)?

- In what ways do you think the arts programme has affected your lifestyle?

- New career paths?

- Creativity?

- Purposeful activity?

- Quality of life? Relationships?

- Can you describe your favourite part of the programme or a special time in the programme that has had a big impact on you?

- Award at an exhibition?

- Acknowledgement/compliment from a peer?

- Development of a certain skill?

- Discussing an art piece that has significance to the artist's recovery process - Can you describe this art piece and when you did it?

- Why is this piece significant in your recovery? 